\title{
Comparison of Lumbopelvic Motions During Hip Medial Rotation Depending on Sex Differences and Chronic Lower Back Pain
}

\author{
ChiHwan Kim', JinTae Han² \\ 'Department of Physical Therapy, Graduate school, Kyungsung University, Busan; ${ }^{2}$ Department of Physical Therapy, College of Science, Kyungsung \\ University, Busan, Korea
}

Purpose: Hip rotation testing is important in the evaluation of chronic back pain. The purpose of this study was to investigate hip and lumbopelvic movement during hip medial rotation (HMR) in individuals with chronic lower back pain (CLBP).

Methods: This study targeted 112 subjects in total: 28 healthy males and 28 healthy females, and 27 males with CLBP and 29 females with CLBP. Motion-capture device was used to measure the hip medial rotation angle (HMRA), lumbopelvic rotation angle (LPRA), and the rotation angle of the hip when lumbopelvic rotation starts during hip medial rotation.

Results: When evaluating the healthy males and females using the hip medial rotation test (HMRT), healthy males showed a smaller HMRA than did healthy females $(p<0.05)$. When evaluating the healthy males and the males with CLBP using the HMRT, males with CLBP showed a smaller HMRA and more lumbopelvic movements than did healthy males $(p<0.05)$ in addition, their lumbopelvic movements occurred earlier during HMR $(p<0.05)$. Finally, when evaluating the males and the females with CLBP using the HMRT, males with CLBP showed a smaller HMRA and more lumbopelvic movements $(p<0.05)$, and their lumbopelvic movements occurred earlier during HMR $(p<0.05)$.

Conclusion: The HMRT is an important test for the evaluation of males, and especially males with CLBP, as they often experience an increased LPRA and decreased HMRA, with lumbopelvic movement occurring earlier during HMR when compared to other groups.

Keywords: Chronic lower back pain, Hip medial rotation, Lumbopelvic movement

\section{INTRODUCTION}

Hip rotation affects lumbopelvic movement and is associated with lower back pain (LBP). ${ }^{1}$ The movement system impairment model (MSI) classifies the direction of the lumbar movement causing LBP. If the symptoms are related to lumbar rotation movement, it is classified as lumbar rotation syndrome. The stiffness of the muscles and joints makes movements to the specific direction of the adjacent other joints, which causes accumulation of micro injuries leading to massive damage. Therefore, in the MSI model, it is suggested that the most effective treatment is to evaluate and treat the contributing factors that cause damage rather than the damage itself, to restore abnormal alignment and inaccurate motions. ${ }^{2,3}$ The hip medial rotation test (HMRT) is an important test in determining whether lumbar rotation syndrome is the cause of LBP. ${ }^{4}$ If lumbopelvic

Received Mar 19, 2019 Revised Apr 25, 2019

Accepted Apr 30, 2019

Corresponding author Jin Tae Han

E-mail jthan2001@ks.ac.kr movement occurs too early during hip rotation in the prone position, the ability to stabilize the lumbar is decreased and tissues associated with limited or stiff hip rotation can overtravel within the lumbopelvic region. These motion defects can cause excessive and frequent lumbopelvic movement during hip rotation, which can eventually cause pain. Various interventions have been able to reduce repetitive lumbopelvic movements, ${ }^{5-7}$ thereby reducing LBP.3.4

A number of studies involving hip rotation have examined sex, age, and lifestyle when selecting subjects. It has been demonstrated that there is a difference based on $\operatorname{sex}^{8}$ in the range of hip rotation and in the HMRT.,10 In the hip rotation angle measurement, the hip medial rotation angle (HMRA) of the males was $44^{\circ}$ to $47^{\circ}$ and the HMRA of the females was $51^{\circ}$ to $54^{\circ}$, the HMRA of the females was larger than that of the males. The hip lateral rotation angle (HLRA) of the males was $52^{\circ}$ to $53^{\circ}$ and the HLRA of the females
Copylight (C)2019 The Korean Society of Physical Therapy

This is an Open Access article distribute under the terms of the Creative Commons Attribution Non-commercial License (Http:// creativecommons.org/license/by-nc/4.o.) which permits unrestricted non-commercial use, distribution, and reproduction in any medium, provided the original work is properly cited. 
was $47^{\circ}$ to $48^{\circ}$, HLRA was larger than that of the females. ${ }^{8}$ In a hip lateral rotation test (HLRT) in a patient with chronic lower back pain (CLBP), lumbopelvic rotation angle (LPRA) of the males with CLBP was $6.1^{\circ}$ and that of the females with CLBP was $4.6^{\circ} .9$ In the HMRT, the LPRA of the males with CLBP was $10.0^{\circ}$ and the female with CLBP was $4.5^{\circ}$. The HMRA of the males with CLBP was $30^{\circ}$ and that of the females with CLBP was $42^{\circ}$. The initiation of pelvic rotation was the males with CLBP was $5.4^{\circ}$ and the females with CLBP was $16.0^{\circ} .^{10} \mathrm{~A}$ difference has also been seen in the flexibility and range of the hip joints between adults and infants, ${ }^{11,12}$ and in the range of movement of the hip joints depending on whether the subject has a lifestyle that involves a lot of sitting or standing. The sitting lifestyle peoples showed a larger hip rotation angle than the standing lifestyle peoples. ${ }^{13}$ The angle of movement used in hip joints should be different for Asian peoples than it is for Western peoples due to their differing sitting styles. Asian peoples have a lot of sitting lifestyle, and Western people have a lot of standing lifestyle. So Asian peoples need a greater hip rotation angle for daily of living. ${ }^{14}$

There have been many studies examining results of the HLRT and HMRT in patients with chronic LBP in relation to sex. ${ }^{6,9,10}$ However, research evaluating hip and pelvic movement in hip medial rotation (HMR) based on sex and LBP is lacking. Therefore, this study aims to investigate the correlation between HMR and lumbopelvic movement depending on sex and LBP.

\section{METHODS}

\section{Subjects}

Patients with CLBP were diagnosed with LBP at the hospital and had complained of LBP for more than 12 weeks; they ranged in age from 20 to 59 years. Subjects who were able to stand or walk without assistance and who had an LBP score of two or higher on the visual analog scale were included in the study. Healthy subjects were those who did not have pelvic or spinal neuromuscular disease and who had not experienced LBP or pelvic pain within the past six months. We excluded all patients with hip or knee joint injuries that limit daily activity, with spinal fractures, with a history of surgery, tumors, or spinal deformities such as scoliosis, with a body mass index (BMI) higher than 30, who were pregnant at the time of the study; who could not accurately express pain due to mental illness; or who had a severe medical condition at the time of the study. The experi- ment was explained thoroughly to the subjects, and they voluntarily agreed to participate in the study.

\section{Experimental methods}

\section{1) Measurement}

MyoMOTION (myoMOTION Research PRO, Noraxon Inc., Germany) is a wireless motion-capture device that uses an inertial measurement unit sensor. The inertial measurement unit provides direction information by combining angular velocity, acceleration, and geomagnetic sensor information. ${ }^{15}$ Motion-capture device unit 1 was placed at the sacrum, while motion-capture device unit 2 was placed at the center of the knee. The slope of motion-capture device unit 1 was measured the LBRA. The angle of rotation of the hip joint was evaluated by measuring the slope of motion-capture device unit 2. Motion-capture device measured the angle of HMR in about 1 millisecond in real time.

\section{2) Experimental procedure}

The medial rotation test measured the HMRA and the LPRA, and the initiation of the pelvic rotation angle was measured during HMR. In an anatomical posture, the rotation movement in the horizontal plane with respect to the vertical axis of the lumbopelvic was defined as a lumbopelvic rotation. Hip joint rotation was defined as horizontal rotation of the hip joint based on the vertical axis of the hip joint. The initiation of lumbopelvic rotation was defined as the

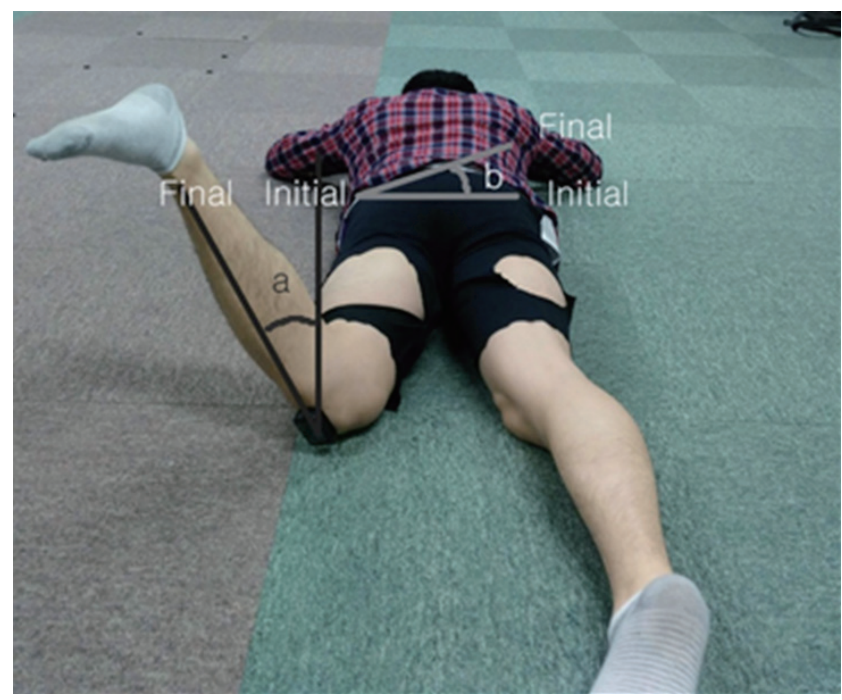

Figure 1. Kinematic model with calculations for hip medial rotation (HMR) and lumbopelvic rotation. (a) Hip medial rotation angle (HMRA) $\left({ }^{\circ}\right)$, (b) lumbopelvic rotation angle (LPRA) $\left(^{\circ}\right)$. 
point when both the angular displacement of the pelvic segment exceeded $0.5^{\circ}$ and the angular velocity of the pelvic segment exceeded $15 \%$ of its maximum (Figure 1).

The subjects were instructed to lie prone, with one knee flexed to $90^{\circ}$ and the hip neutral with regard to rotation and abduction/adduction. They were then instructed to actively medially rotate their hip as far as possible and return to the starting position. In prone position, HMR occurs as the lower leg and foot rotate away from the midline. The subjects were given up to 10 seconds to complete the full movement. ${ }^{9}$ The test was performed on dominant legs.

\section{3) Visual analog scale}

Pain intensity was measured using the visual analogue scale (VAS). On the $10 \mathrm{~cm}$ line where the scale was not drawn on the questionnaire, the patient was instructed to indicate the degree of back pain of the patient to the point, and the distance to the point was measured to one digit below the decimal point. ${ }^{16}$

\section{Statistical analysis method}

Multiple comparisons between groups were determined by two-way ANOVA. An independent t-test was used to compare each variable between the groups. Data are presented as mean \pm standard deviation (SD). Statistical analysis was performed using SPSS Version 24.0
(SPSS Inc., Chicago, IL, USA). The significance level, ( $\alpha$ ) was 0.05.

\section{Research ethics approval}

This study was approved by the Institutional Review Board of Kyungsung University in 2016, and the approval number is KSU-16-12-10-001.

\section{RESULTS}

\section{General subject characteristics}

In this study, a total of 112 patients were included: 28 healthy males, 28 healthy females, 27 CLBP male, and 29 CLBP female patients. Because of sex differences, there were statistically significant differences in height, weight, and BMI between the groups $(\mathrm{p}<0.05)$. However, there were no statistically significant differences in age, VAS or CLBP onset between the groups $(\mathrm{p}>0.05)$. The mean \pm standard deviation values are presented in Table 1.

\section{Comparison of lumbopelvic movement during HMRT depending on sex and CLBP}

Result of two-way ANOVA, LPRA of male was significant larger than that of female, HMRA of male was significant smaller than that of female, and HMRA when lumbopelvic rotation begins of male was significant smaller than that of female $(\mathrm{p}<0.05)$. LPRA of CLBP group was significant larger than that of healthy group,

Table 1. General characteristics of the study subjects

\begin{tabular}{|c|c|c|c|c|}
\hline \multirow{2}{*}{ Variable } & \multicolumn{2}{|c|}{ Healthy Group } & \multicolumn{2}{|c|}{ CLBP Group } \\
\hline & Male $(n=28)$ & Female $(n=28)$ & Male $(n=27)$ & Female $(n=29)$ \\
\hline Age (yr) & $39.00 \pm 9.69$ & $38.57 \pm 10.69$ & $40.11 \pm 9.71$ & $39.97 \pm 10.99$ \\
\hline Height (cm) & $172.86 \pm 4.23$ & $158.82 \pm 6.93$ & $174.07 \pm 5.37$ & $157.79 \pm 6.00$ \\
\hline Weight (kg) & $72.39 \pm 7.64$ & $55.18 \pm 5.85$ & $73.96 \pm 6.95$ & $55.90 \pm 5.91$ \\
\hline $\mathrm{BMI}\left(\mathrm{kg} / \mathrm{m}^{2}\right)$ & $24.20 \pm 2.22$ & $21.85 \pm 1.70$ & $24.42 \pm 2.16$ & $22.47 \pm 2.16$ \\
\hline VAS (score) & & & $5.20 \pm 1.22$ & $4.76 \pm 1.51$ \\
\hline LBP onset (weeks) & & & $32.59 \pm 14.25$ & $27.21 \pm 11.37$ \\
\hline
\end{tabular}

Mean \pm SD

CLBP: chronic lower back pain, BMl: body mass index, VAS: visual analog scale.

Table 2. Comparison of lumbopelvic movement between with and without CLBP based on sex

(unit: $\left.{ }^{\circ}\right)$

\begin{tabular}{|c|c|c|c|c|}
\hline \multirow{2}{*}{ Movement } & \multicolumn{2}{|c|}{ Male Group } & \multicolumn{2}{|c|}{ Female Group } \\
\hline & Healthy $(n=28)$ & $\operatorname{CLBP}(n=27)$ & Healthy $(n=28)$ & $\operatorname{CLBP}(n=29)$ \\
\hline Active LPRA $\left({ }^{\circ}\right)$ & $5.34 \pm 2.55$ & $8.81 \pm 3.13^{\neq}$ & $4.52 \pm 2.57^{*}$ & $5.05 \pm 1.63^{\S}$ \\
\hline Active HMRA $\left({ }^{\circ}\right)$ & $34.75 \pm 8.69$ & $27.72 \pm 7.82^{+}$ & $42.29 \pm 9.90^{*+}$ & $45.90 \pm 8.33^{\S}$ \\
\hline HMRA when lumbopelvic rotation begins $\left({ }^{\circ}\right)$ & $10.88 \pm 6.18$ & $6.18 \pm 4.30^{\ddagger}$ & $11.70 \pm 5.79^{*}$ & $14.88 \pm 7.02^{\S}$ \\
\hline
\end{tabular}

Mean \pm SD

CLBP: chronic lower back pain, LPRA: lumbopelvic rotation angle, HMRA: hip medial rotation angle.

${ }^{*} p<0.05$ significant difference between male and female; ${ }^{t} p<0.05$ significant difference between CLBP group and healthy group (by two-way ANOVA); $p<0.05$ significant difference between healthy male and male with CLBP; ${ }^{\mathrm{p}}<0.05$ significant difference between male with CLBP and female with CLBP (by independent t-test). 
HMRA of CLBP group was significant smaller than that of healthy group, and HMRA when lumbopelvic rotation begins of CLBP group was significant smaller than that of healthy group $(\mathrm{p}<0.05)$ (Table 2). Also, results of the two-way ANOVA showed significant interactions (sex $\times$ CLBP) in HMRA, LPRA, HMRA when lumbopelvic rotation begins $(\mathrm{p}<0.05)$. In other words, in male with CBLP, LPRA was increased in female, while HMRA and HMRA when lumbopelvic rotation bigins was decreased. When comparing healthy males and healthy females using the HMRT, healthy males showed a smaller HMRA than did healthy females $(\mathrm{p}<0.05)$. When comparing the males and the females with CLBP using the HMRT, males with CLBP showed a smaller HMRA and more lumbopelvic movements than the females with CLBP $(\mathrm{p}<0.05)$ further, their lumbopelvic movements occurred earlier during HMR $(\mathrm{p}<0.05)$. When comparing the healthy males and the males with CLBP using the HMRT, the males with CLBP showed a smaller HMRA and more lumbopelvic movements than did the healthy males $(\mathrm{p}<0.05)$ further, their lumbopelvic movements occurred earlier during HMR $(\mathrm{p}<0.05)$. The mean \pm standard deviation values are presented in Table 2.

\section{DISCUSSION}

This study measured the HMRA, LPRA, and rotation angle of the hip when lumbopelvic rotation begins using the HMRT. The subjects were classified according to sex and the presence of CLBP. When comparing healthy males and healthy females using the HMRT, healthy males showed a smaller HMRA than did healthy females. Kim et al. ${ }^{13}$ found that hip lateral rotation of Asian peoples was usually larger than that of Western peoples when they sit crossed-leg on the floor because sitting on the floor are common daily living in Asian peoples. Han et al. ${ }^{8}$ evaluated the angle of rotation of the hip joint of Japanese males who frequently sit crossed-leg. And they found that sitting crossed-leg cause to increase the hip lateral rotation (HLR), because it induced the stiffness and shortening of hip medial rotator. Braten et al. ${ }^{17}$ reported that the HMRA decreases and the hip lateral rotation angle increases due to a reduced femoral torsion. Therefore, it is suggested that the limitation of the HMR in males is due to a difference in the femoral torsion, posture change, and the related muscles.

When comparing the healthy males and the males with CLBP using the HMRT, males with CLBP showed a smaller HMRA and more lumbopelvic movements than did healthy males, further, their lumbopelvic movements occurred earlier during HMR. As such, treatment emphasizing the lumbopelvic stability of patients with CLBP could reduce the amount of excessive lumbopelvic movement and prevent lumbopelvic movement early during hip joint rotation. ${ }^{5}$ It has been found that it is possible to reduce LBP by reducing the movement of the lumbopelvic during lower extremity movement. ${ }^{3,4}$ Male with CLBP are less able to stabilize the lumbopelvic during lower extremity movements and are believed to have limited movement as a result due to stiffness and the shortening of tissues associated with HMR.

When comparing the healthy females and the females with CLBP using the HMRT, there was no significant difference. Hoffman et al. ${ }^{10}$ reported that a result of a study in which males and females were divided into patients with CLBP. However, there has been no study of the HMRT comparing healthy females with a females with CLBP. In previous studies, the HMRT showed a significant difference in the stability of the lumbar spine associated with CLBP and the limitation of movement of the hip movement according to gender. ${ }^{10,17}$ The females was not affected by the hip medial rotation, so it did not affect the hip medial rotation test in the females with CLBP. In the HLRT, females with CLBP are more restricted to HLR than healthy females, There was a significant difference in the HLRT. ${ }^{18}$

When comparing the males and females with CLBP using the HMRT, males with CLBP showed a smaller HMRA and more lumbopelvic movements; further, their lumbopelvic movements occurred earlier during HMR. Lee et al. ${ }^{19}$ reported that the angle of HMRA was lower in males with CLBP and that the HMRA was higher in females with CLBP. Hoffman et al. ${ }^{6}$ showed similar results in current study. Males with CLBP had a greater LPRA than females with CLBP. And Males with CLBP had lumbopelvic movements occurred earlier during HMR than females with CLBP. These results may be attributed to the limitation of the hip rotation angle which, as mentioned above, is based on sex and the decrease in the lumbopelvic stabilization ability of LBP patients.

Some limitations of the HMRT include that it is not a functional test, that it has not been studied in relation to the muscles involved, and that it has not been associated with. In the future, the relationship between the mobilization pattern, strength, length, and stiffness of the involved muscle according to sex and CLBP in the 
HMRT should be investigated, as well as whether there is a difference in the movement of the hip joint between Asian and Western populations. It is also necessary to investigate the therapeutic intervention methods required to improve lumbopelvic movement when hip rotation is applied in patients with CLBP.

\section{CONCLUSION}

When comparing healthy males and healthy females using the HMRT, healthy males showed a smaller HMRA than did healthy females. When comparing the healthy males and the males with CLBP using the HMRT, males with CLBP showed a smaller HMRA and more lumbopelvic movements than did healthy males; further, their lumbopelvic movements occurred earlier during HMR. When comparing the males and females with CLBP using the HMRT, males with CLBP showed a smaller HMRA and more lumbopelvic movements; further, their lumbopelvic movements occurred earlier during HMR.

In this study, males should be carefully evaluated in the HMRT. In addition, we found that improvement of lumbopelvic rotation movements associated with HMR was necessary for males with CLBP.

\section{REFERENCES}

1. Harris-Hayes M, Sahrmann SA, Van Dillen LR. Relationship between the hip and low back pain in athletes who participate in rotation-related sports. J Sport Rehabil. 2009;18(1):60-75.

2. Sahrmann SA. Diagnosis and treatment of movement impairment syndromes, 3th edition, seoul, jungdam media, 2005;79-151.

3. Van Dillen LR, Sahrmann SA, Wagner JM. Classification, intervention, and outcomes for a person with lumbar rotation with flexion syndrome. Physical therapy. 2005;85(4):336-51.

4. Van Dillen LR, Maluf KS, Sahrmann SA. Further examination of modifying patient-preferred movement and alignment strategies in patients with low back pain during symptomatic tests. Man Ther. 2009;14(1):52-
60.

5. Hoffman SL, Johnson MB, Zou D et al. Effect of classification-specific treatment on lumbopelvic motion during hip rotation in people with low back pain. Man Ther. 2011;16(4):344-50.

6. Hoffman SL, Johnson MB, Zou D et al. Gender differences in modifying lumbopelvic motion during hip medial rotation in people with low back pain. Rehabil Res Prat. 2012;2012.

7. Scholtes SA, Norton BJ, Lang CE et al. The effect of within-session instruction on lumbopelvic motion during a lower limb movement in people with and people without low back pain. Man Ther. 2010;15(5): 496-501.

8. Han H, Kubo A, Kurosawa K et al. Hip rotation range of motion in sitting and prone positions in healthy japanese adults. J Phys Ther Sci. 2015;27(2):441-5.

9. Gombatto SP, Collins DR, Sahrmann SA et al. Gender differences in pattern of hip and lumbopelvic rotation in people with low back pain. Clin Biomech. 2006;21(3):263-71.

10. Hoffman SL, Johnson MB, Zou D et al. Sex differences in lumbopelvic movement patterns during hip medial rotation in people with chronic low back pain. Arch Phys Med Rehabil. 2011;92(7):1053-9.

11. Phelps E, Smith LJ, Hallum A. Normal ranges of hip motion of infants between nine and 24 months of age. Developmental Medicine \& Child Neurology. 1985;27(6):785-92.

12. Sankar WN, Laird CT, Baldwin KD. Hip range of motion in children: what is the norm? J Pediatr Orhop. 2012;32(4):399-405.

13. Kim RB, Jo JH, Kim SJ. The effects of oriental sedentary and western sand-up meal life style on gait. Journal of Sports and Leisure Stuies. 2016; 63:683-90.

14. Mulholland SJ, Wyss UP. Activities of daily living in non-western cultures: range of motion requirements for hip and knee joint implants. Int J Rehabil Res. 2001;24(3):191-8.

15. Saber-Sheikh K, Bryant EC, Glazzard C et al. Feasibility of using inertial sensors to assess human movement. Man Ther. 2010;15(1):122-5.

16. Wang JM, Kim DJ. Assessment of the spinal pain using visual analogue. Korean Society of Spine Surgery. 1995;2(2):177-84.

17. Bråten M, Terjesen T, Rossvoll I. Femoral anteversion in normal adults: ultrasound measurements in 50 men and 50 women. Acta Orthop Scand. 1992;63(1):29-32.

18. Kim CH, Han JT. Comparison of hip and lumbopelvic movement while hip lateral rotating in individual with chronic low back pain. J Kor Phys Ther. 2017;29(5):241-5.

19. Lee JY, Han SY, Nam HW et al. Comparison of hip internal rotation angle in chronic low back pain patients according to the gender. The Journal of Korea CHUNA Manual Medicine for Spine \& Nerves. 2010;5(2):9-16. 